\title{
Commission 50 Report: Identification and Protection of Observatory Sites.
}

\section{Introduction}

Fundamental to all observational astronomy, whatever the technique or the objects of observation, is the existence of an observing site. Wavelength regions range from the shortest (highest energy) through the optical, IR, millimeter, to the radio region, locations may be anywhere on earth for groundbased (or below ground even) observatories and in space. Certainly the identification of the best sites for observing is a critical item. Naturally, the observing includes both nighttime and daytime conditions. The scientific studies must, of course, include the identification of the best criteria for establishing the merit of a site and of the techniques to assess these criteria. Factors such as the properties of the atmosphere (clear skies, low humidity, low wind, low thermal turbulence, and so forth) and of other such items as site access, the operational aspects of an observatory located at the site, and other such items are important.

In addition, knowledge of many of these items can and will help improve the quality of observations at many existing sites, as we gain knowledge of how to improve astronomical seeing and the efficiency of observing.

An important item, growing in importance with time, is the protection of such observing sites, existing or potential. Light pollution, radio interference, and other such adverse environmental impacts on observations can and do compromise the quality (and even the existence) of our data. Astronomy must address these issues, and work to implement solutions to them.

The role of the Commission is to help all member nations (and observatories and individuals) become aware of these issues, and to foster the necessary communications between the various astronomy interests and those outside of astronomy that are also adversely affected or who can help in ameliorating the problems.

These problems where addressed at IAU Colloquium No. 112, held immediately after the last General Assembly meeting. A short report on the meeting follows:

Brief Summary of the Colloquium: There were approximately 120 attendees (about 80 from the U.S.A.), and 78 papers were presented in the three and a half days of meetings. The attendees came from 19 countries world-wide.In addition to the attendees, representatives of the media were in attendance, and there was a formal press conferences as well as numerous interviews with attendees and speakers. The meeting was cosponsored by the CIE (Commission Internationale de l'Eclairage), COSPAR (Committee on Space Research), and URSI (Union Radio Scientifique Internationale).

It is clear from the papers presented that observatories around the world and on satellites in space are in danger from the proliferation of outdoor lighting, increased radio frequency interference, and from the space junk generated as satellites reach the ends of their operating lifetimes and are abandoned. Abandoned satellites often break up and are a source of debris that can damage orbiting telescopes or other satellites. Every photograph taken with the 48-inch Schmidt telescope at Mt. Palomar Observatory in southern California or with the similar telescope at Anglo-Australian Observatory in Australia is marred by the streaks from satellites or space junk.

Numerous speakers addressed the question of light pollution, as it affects astronomy and the general public, discussing how it is mainly due to poor quality lighting. 
There are solutions to the problem, such as using the correct amount of light for the task, not overkill, using quality, well shielded fixtures, using time controls when possible, and using low pressure sodium light sources whenever feasible. All of these solutions also make for better quality lighting and save money, rather than costing more money. Several speakers were from the lighting industry, and their talks reflected the same concerns and solutions.

The urban sky glow at some observatories is now of such a high level that closures are considered. The 100-inch telescope at Mt. Wilson Observatory, near Los Angeles, has been closed. Mt. Palomar Observatory, with the largest present telescope in the United States, suffers from a sky glow level twice the natural background. Even observing sites with presently dark skies will be seriously threatened over the next few decades.

Few members of the general public have even seen a prime dark sky, as urban sky glow has removed the view of the universe that their ancestors had only a generation or two ago. Mankind faces the specter of being only able to see the universe on TV or on a planetarium in only a few generations, unless awareness of the problem grows and solutions are implemented.

With respect to the space debris issue, the amount of space debris already exceeds the amount of meteoroids, and the amount of space junk is growing with time. Much of the space junk is permanently in orbit, though that in low orbit re-enters the atmosphere and is destroyed (only large fragments survive to fall in the sea or on land). A $3 \mathrm{~mm}$ diameter piece of space junk has the same kinetic energy has a bowling ball going 60 miles per hours. It can be lethal to operating space satellites, and their contents.

At present, only 5 percent of the objects in space larger than a meter or so in diameter are operating space satellites; the rest are spent vehicles or space debris. By the 1990 s or the year 2000 , the debris population will become so large that collisions among the debris will be frequent, causing more and more fragments and more and more collisions. In less than 100 years, it is likely, that without active and effective countermeasures, new satellites launched into space will collide with debris and destruct in an extremely short time, thus making space unusable for science or for the benefit of mankind.

NASA, the European Space Agency, and others with an active interest in operating in the space environment are beginning to become aware of the serious nature of the issue, and studies are underway about how best to cope with the problem. It is not only astronomy who is and will be serious bothered by the issue.

Astronomers, and others, are particularly worried by what they perceive as frivolous uses of space, such as for advertising, for "burial" of cremated bodies, or for any application that does not benefit all of mankind. Only a few years ago, people were not very aware of space pollution as a problem, just as not many years ago people had no conception that the atmosphere or the oceans could become so polluted in such a short time. Now we are beginning to realize that space too is finite, and that it is beginning to fill up with trash.

The situation with radio frequency interference effects on astronomy is in many ways the most serious at the present time. While there are international committees that regulate the assignment of radio frequencies, the commercial and other pressures for the use of such frequencies is so high that it is becoming extremely difficult to reserve some of the frequencies most important for radio astronomy as "radio quiet bands." Just as some of 
the prime land areas of the earth need preservation as "wilderness areas," some of the radio frequencies need preserved as protected bands.

A special threat to radio astronomy are the transmitters on space satellites, as their transmissions literally cover the earth, and there is no possibility of locating an observing site well away from transmission stations. Some of the critical frequencies for important work in radio astronomy have already been lost. As with the issues of light pollution and space debris, the RFI problem is truly a global one. Solutions to some aspects of the problem are possible, but require added costs for such things as filters. These added costs are well justified, in the view of astronomers, to preserve our windows to the universe.

Several papers at the meeting discussed other adverse environmental issues affecting astronomy. Geophysical and astronomical sites are affected by air pollution, vibrations, and thermal pollution as well as the issues mentioned above. For example, the US Naval Observatory in Washington DC has just been through a detailed study of the potential threat to the critical work done there; added thermal turbulence over the site by the construction of a large heated building close by was shown to compromise greatly the astrometric character of the site.

In summary, all attendees believed strongly that astronomy, and, indeed, all of mankind, are rapidly losing their view of the universe. This insensitivity to the environment, whether due to light pollution, RFI, or increasing space debris, is similar to the environmental impacts due to air pollution, water pollution, toxic waste, and other threats. Mankind must increase its awareness to these critical issues, and develop and implement solutions. Astronomy and the public deserve a view of the universe.

Outline of the issues, as presented at the meeting:

* Most existing observatories are already suffering from light pollution, radio interference, or effects of space debris.

* The environmental threat to observatories is rapidly increasing. Some aspects of astronomy are probably doomed.

* Observatories in space, built at great cost, may be seriously affected by orbital debris or other man-made space pollution.

* All planning of future observatories on earth and in space must take environmental deterioration into account.

* The human experience of the inspiring beauty of the cosmos is at risk for all people, not just scientists, as light pollution destroys our view of the beautiful dark sky.

The proceedings are in press at the time of the writing of this report (Sep. 1990).

Another rich resource for those interested in the topics addressed by Commission 50 is the Astronomy and Astrophysics Abstracts volumes, issued twice a year. The section of the these that includes many papers about site testing is \# 082 , which deals with the atmosphere (refraction, scintillation, extinction, and site testing). Other sections may well contain other papers of interest, and one should carefully explore these most valuable volumes for papers that address problems of interest. It is perhaps especially important for topics related to the issues that Commission $\mathbf{5 0}$ deals with, as some of the relevant papers are published in "non-astronomy" journals.

During the years covered by this report, there were many papers of interest published, dealing with worldwide observing sites, including those in the U.S.A., the 
USSR, China, India, Turkey, Chile, and Antarctica. We will not mention any specifically here: refer to the AAA volumes. Many papers also addressed the general questions of criteria established to recognize and evaluate quality observing sites.

Certainly, progress in these matters has been good, and we are now understanding much better the criteria for understanding our observing sites (and how to build them without degrading them) and how to select new ones. The result is that the quality of some of the site surveys done recently (or still underway) is much better than previous ones. Attention will still need to made to preserve the quality of the best observing sites so that they remain viable and production for the indefinite future.

\section{Specific Activities Reported on by Commission Members.}

J.-P. Swings reports on the progress of the ESO VLT sites studies in Chile. This is undoubtedly the most extensive and comprehensive site study that has been done.

(Besides the scientific characteristics of the sites, they are also looking into the managerial, operational, and financial problems linked to the selection of a site. These are certainly also important items relative to an effective site study!) The Working Group has essentially made a selection between two areas that were preselected in earlier studies. The Paranal area has turned out to be the best on the basis of the studies, and astronomically superior to the La Silla area in terms of the number of photometric nights, the relative humidity, the precipitable water vapour, and, marginally, the seeing, light pollution, and the wind speed at the tropopause. Details are available from ESO.

J.-P. Swings also reports that the European Space Agency has established a Space Debris Advisory Group (SDAG), which will advise in all matters related to space debris and will review the ESA research activities related to space debris. It can take up space debris issues which it deems important. It will provide a European forum for exchange of information on space debris matters and a means to inform on and possibly harmonize national space debris related activities. It will be composed of experts from the Member States having an active involvement in some aspect of space debris. J.-P. Swings is a member of the SDAG, and further details can be obtained directly from him.

The SDAG research program will address the following main areas: environment definition, risk assessment, and protective measures. The definition programs include: analysis of available IRAS data, analysis of material returned from space, dust and debris collection experiments, suitability of optical sensors and radar sensors, and reference population models and future evolution. The risk analysis is currently needed in two orbital regions: LEO (Low Earth Orbit) and GEO (Geostationary Orbit), as the growth of the debris population (the flux of natural objects exceeds the flux of man made objects only in the 0.1 to 1 millimeter range) means that the probability of collision is above acceptable levels in some regions of space. Protective measures are already now required. Generally they are active, aiming for a reduction of the debris population, or passive, protecting objects in space or on the ground. Such measures include: minimization of debris creation, de-orbiting into the lower atmosphere, re-orbiting of satellites at end-oflife into a disposal orbit, debris collection and return to Earth, shielding of space vehicles, and selection of collision free trajectories.

Y. Kozai reports that the astronomical environment around any optical observatory in Japan is much worse than any IAU standard, as any place under good weather is not far from several large cities. Still, every observatory in Japan has regular meetings with senior 
officials of the nearby cities to try to keep the environment from getting any worse.

J. Galt continues to monitor and report on the contamination effects of satellites on radio astronomy. He notes that the problem of pollution of radio astronomy bands is already serious, and, in particular, that the establishment of the GLONASS system has severely curtailed radio studies of important classes of stars.

M. Pande reported on a site evaluation program, for installation of a large telescope, at Chaukori $(2060 \mathrm{~m})$, Devsthal $(2360 \mathrm{~m})$, Gananath $(2060 \mathrm{~m})$, and Mornaula $(2270 \mathrm{~m})$ in the Kumaon range of Siwalik Ranges in Uttar Pradesh, India. The average number of photometric nights (clear more than 4 consecutive hours) in a year are found to be 180 at Dhaukori, 200 at Devsthal, 190 at Gananath, and 220 at Mornaula. The astronomical seeing an microthermal fluctuation observations were carried out at Devsthal. The seeing as estimated from polar trails is less than 1 arc sec for $40 \%$ of the clear nights. Microthermal fluctuations at a height of $24 \mathrm{~m}$ above ground were found to be less than $0.01 \mathrm{deg} C$.

$\mathrm{V}$. Blanco notes that there is now a local ordinance prohibiting future obnoxious outdoor lighting in the cities of La Serena, Coquimbo, Vicuna, and Andacollo, near the Cerro Tololo Interamerican Observatory. He notes that the ESO NTT telescope achieved 0.39 arc sec imagery and that only about 0.25 arc sec was attributable to atmospheric effects, the remainder being due to instrumental effects, concluding that it is vitally important to preserve the best sites for future observing, including those in Chile and in Hawaii.

A. Kubicela unfortunately notes that Belgrade astronomy is not now in a position to locate and build a new observatory. They hope to continue their interest in a future observatory in good observing conditions.

E. Leibowitz reports that the observing conditions at the Wise Observatory site in the Negev desert of southern Israel has not changed much in recent years. The difficulties they had with local authorities relative to the establishment of a nearby ranch (1 km away) were resolved with the ranch being removed to another location where its effects on the quality of the night sky are negligible. The input by Commission 50 was most helpful. The worst polluter of their sky is the town of Mitzpe Ramon, who halo of light dominates the eastern sky. There are difficulties with lighting controls in this city, though the resolutions of the IAU have been helpful in discussions. Fortunately, the zenith sky has not brightened very much is the last 10 years. Current numbers are $\mathrm{U}=20.8, \mathrm{~B}=21.5, \mathrm{~V}=$ $20.9, R=20.7$, and $I=20.2 \mathrm{mag}$ per square arc sec.

G. Swarup notes that he expects that apart from the general increase in radio noise levels that he expects the biggest threat to radio astronomy will be from spread spectrum techniques. He urges serious discussion about this potential, with the involvement of Commission 40.

T. Spoelstra and H. Kahlmann were involved in the European Science Foundation Committee on Radio Astronomy Frequencies (CRAF). Kahlmann is its chairman. the mandate for CRAF is "Coordinating in Europe the radio astronomers' case for discussion with the big public and private telecommunications agencies." It also acts as a European voice to join with radio astronomers globally in the efforts made within the international bodies that allocate frequencies. It initiates and encourages scientific studies aimed at reducing interference. In the period 1987 to July 1990, CRAF had six meetings. 
T. Spoelstra worked with Yang Yi-pei on the development of software methods to reduce interference in observations with synthesis radio telescopes. A method based on interference rejection in the time domain and one based on filtering in the frequency domain gives some promising results.

J. Temko, with others in Czechoslovakia, are continuing to monitor the long term atmospheric transparency variations using observations of standard stars. They have finished a search for a new observatory site, with results of the study archived at the Astronomical Institute in Tatranska Lomnica. They are finding only a few small successes with the battles against light pollution.

D. Crawford reports as follows: Outdoor lighting ordinances continue to be enacted throughout southwestern U.S.A., for the island of Hawaii, and elsewhere where locals have been active in attempting to protect observatories. There are now over 50 such ordinances in Arizona, for example. These ordinances are being effective in protecting observing conditions; for example, the sky glow above Kitt Peak is presently three or four times lower than one would estimate it would be without the ordinances.

A non-profit organization, the International Dark-Sky Association, has been incorporated, with the goals of building awareness of the issues and of the solutions to the problems. So far, it has concentrated on the light pollution issue, but will expand its efforts to all the topics of interest to Commission 50. As of Sep. 1990, IDA has grown to over 600 members, from 44 states in the U.S.A. and from 25 other countries. There are 74 organizational members, including a number of universities and observatories, lighting companies, and many amateur astronomy clubs or societies. Individual memberships begin at $\$ 20$ (organizational ones at $\$ 100$ ). Close to $28 \%$ of those who have joined so far have done so at more than the standard rate. Renewal are running close to $90 \%$. There will be local Sections established to support local efforts. Several have already begun activities. We hope and expect that IDA will grow to become a viable and effective force locally and internationally in behalf of preserving mankind's view of the universe. All members of the IAU interested in the issues (everyone, we hope) are welcome and encouraged to join. We need help to insure a viable future for astronomical research. 